\title{
INTEGRAL REPRESENTATION OF MULTIPLICATIVE, INVOLUTION PRESERVING OPERATORS IN $\mathfrak{L}\left(C_{0}(S, A), B\right)$
}

\author{
STEPHEN T. L. CHOY
}

\begin{abstract}
Bounded linear operators from the space of continuous vector-valued functions which preserve multiplication and involution are characterized in terms of their representing measures. A key role is played by the Arens product in the second dual of a Banach algebra.
\end{abstract}

1. Introduction. Let $S$ be a Hausdorff topological space and let $A, B$ be locally convex Hausdorff topological vector spaces over the real or complex field. Denote by $C(S, A)$ the space of continuous functions from $S$ to $A$ and let $C_{0}(S, A)$ be the subspace of continuous functions vanishing at infinity. If $A=C$, the set of all complex numbers, we simply write $C(S)$ or $C_{0}(S)$. Let $T$ be a bounded linear operator from $C(S, A)$ or $C_{0}(S, A)$ to $B$. Riesz type representation theorems for $T$ have been studied by numerous authors, for example, see Bartle, Dunford and Schwartz [2], Dinculeanu [6], J. Gil de Lamadrid [7], Goodrich [8], Brooks and Lewis [4] and the author [5]. Now if $A, B$ are Banach algebras with involution and $T$ preserves multiplication and involution, it is natural to ask how these properties are reflected in the representing measure $K$. It was G. W. Johnson [10] who first solved this problem for the case where $S$ is compact, $A=C$ and $B$, as a Banach space, is the dual of another Banach space.

In this paper, by using the Arens product in the second dual of a Banach algebra, we solve this problem in a more general setting. A convenient reference for the Arens product could be found in Bonsall and Duncan [3]. The reader is referred to $[3, \S 12]$ for details of notation and terminology concerning the Arens product not explained here.

In the remainder of this paper $S$ is a locally compact Hausdorff space, $A$ and $B$ are Banach algebras, and $T$ is a bounded linear operator from $C_{0}(S, A)$ to $B$; where $C_{0}(S, A)$ is endowed with the topology of uniform convergence. Let $C_{0}(S)$ $\hat{\otimes} A$ be the completion of the algebraic tensor product $C_{0}(S) \otimes A$ with respect to the least cross norm. Then $C_{0}(S) \hat{\otimes} A=C_{0}(S, A)$, where $C_{0}(S, A)$ is endowed with the uniform norm and the equation indicates isometry between the two spaces (see, for example, [7]). Given $f^{\prime} \in C_{0}^{\prime}(S, A)$ and $x \in A$, the map $f \rightarrow f^{\prime}(f \cdot x)$ for $f \in C_{0}(S)$ is an element of $C_{0}^{\prime}(S)$. Hence there is a unique regular Borel measure $\mu\left(x, f^{\prime}\right)$ such that $\int f d \mu\left(x, f^{\prime}\right)=f^{\prime}(f \cdot x)$ for $f \in C_{0}(S)$. Now given $e$ in $\mathscr{B}(S)$, the

Received by the editors July 21, 1980 and, in revised form, January 19, 1981.

1980 Mathematics Subject Classification. Primary 47B38; Secondary 46G10.

Key words and phrases. Vector-valued measure, multiplicative and involution preserving operators, representing measure, Arens product. 
Borel class of $S$, and $x \in A, 1_{e} \otimes x$ can be viewed as an element of $C_{0}^{\prime \prime}(S, A)$ : $\left(1_{e} \otimes x\right)\left(f^{\prime}\right)=\mu\left(x, f^{\prime}\right)(e)$. Brooks and Lewis [4] show that for each bounded linear operator $T$ from $C_{0}(S, A)$ to $B$ there is a unique weakly regular set function $K$ from $\mathscr{B}(S)$ to $\mathfrak{L}\left[A, B^{\prime \prime}\right]$ so that $T(f)=\int f d K$; in fact, $K(e) x=T^{\prime \prime}\left(1_{e} \otimes x\right)$.

Throughout this paper multiplication in the second dual of a Banach algebra is defined by the Arens product. The second dual of a Banach algebra is again a Banach algebra under the Arens product [3]. I am greatly indebted to the referee for this helpful comments on the presentation of this paper.

2. Multiplicative operators. In this section we obtain a representation theorem for multiplicative operators in the following

TheOREM 2.1. The operator $T$ satisfies $T(f g)=T(f) T(g)$ for all $f, g \in C_{0}(S, A)$ iff the representing measure $K$ satisfies $K\left(e_{1} \cap e_{2}\right)(x y)=\left(K\left(e_{1}\right) x\right)\left(K\left(e_{2}\right) y\right)$ for all $x, y \in A$ and $e_{1}, e_{2} \in \mathscr{B}(S)$.

Before we can prove the theorem we need the following two results:

Proposition 2.2. Let $A, B$ be Banach algebras and let $T: A \rightarrow B$ be a bounded linear operator. Then the operator $T$ satisfies $T(f g)=T(f) T(g)$ for all $f, g \in A$ iff $T^{\prime \prime}(F G)=T^{\prime \prime}(F) T^{\prime \prime}(G)$ for all $F, G \in A^{\prime \prime}$ where $T^{\prime \prime}$ is the second adjoint of $T$.

Proof. Since $A \subseteq A^{\prime \prime}$ all we have to do is to prove the necessity. For every $b^{\prime} \in B^{\prime}$,

$$
\begin{aligned}
T^{\prime \prime}(F G)\left(b^{\prime}\right) & =(F G)\left(T^{\prime}\left(b^{\prime}\right)\right)=F\left(\left[G, T^{\prime}\left(b^{\prime}\right)\right]\right), \\
\left(T^{\prime \prime}(F) T^{\prime \prime}(G)\left(b^{\prime}\right)\right) & =T^{\prime \prime}(F)\left(\left[T^{\prime \prime}(G), b^{\prime}\right]\right)=F\left(T^{\prime}\left[T^{\prime \prime}(G), b^{\prime}\right]\right) .
\end{aligned}
$$

For $f \in A$,

$$
\begin{aligned}
{\left[G, T^{\prime}\left(b^{\prime}\right)\right](f) } & =G\left(\left\langle T^{\prime}\left(b^{\prime}\right), f\right\rangle\right), \\
T^{\prime}\left[T^{\prime \prime}(G), b^{\prime}\right](f) & =\left[T^{\prime \prime}(G), b^{\prime}\right](T(f)) \\
& =T^{\prime \prime}(G)\left(\left\langle b^{\prime}, T(f)\right\rangle\right)=G\left(T^{\prime}\left\langle b^{\prime}, T(f)\right\rangle\right) .
\end{aligned}
$$

For $g \in A$,

$$
\begin{aligned}
\left\langle T^{\prime}\left(b^{\prime}\right), f\right\rangle(g) & =T^{\prime}\left(b^{\prime}\right)(f g)=b^{\prime}(T(f g)), \\
T^{\prime}\left\langle b^{\prime}, T(f)\right\rangle(g) & =\left\langle b^{\prime}, T(f)\right\rangle(T(g))=b^{\prime}(T(f) T(g)) \\
& =b^{\prime}(T(f g)) .
\end{aligned}
$$

We conclude that $T^{\prime \prime}(F G)=T^{\prime \prime}(F) T^{\prime \prime}(G)$.

LEMMA 2.3. For $e_{1}, e_{2} \in \mathscr{B}(S), x, y \in A,\left(1_{e_{1}} \otimes x\right)\left(1_{e_{2}} \otimes y\right)=1_{e_{1} \cap e_{2}} \otimes x y$.

Proof. For $g^{\prime} \in C_{0}^{\prime}(S, A)$,

$$
\begin{aligned}
\left(\left(1_{e_{1}} \otimes x\right)\left(1_{e_{2}} \otimes y\right)\right)\left(g^{\prime}\right) & =\left(1_{e_{1}} \otimes x\right)\left(\left[\left(1_{e_{2}} \otimes y\right), g^{\prime}\right]\right) \\
& =\mu\left(x,\left[\left(1_{e_{2}} \otimes y\right), g^{\prime}\right]\right)\left(e_{1}\right), \\
\left(1_{e_{1} \cap e_{2}} \otimes x y\right)\left(g^{\prime}\right) & =\mu\left(x y, g^{\prime}\right)\left(e_{1} \cap e_{2}\right)=\nu\left(x y, g^{\prime}\right)\left(e_{1}\right) ;
\end{aligned}
$$

where $\nu\left(x y, g^{\prime}\right)(e)=\int_{e} x_{e_{2}} d \mu\left(x y, g^{\prime}\right)$, and $x_{e}$ is the characteristic function of $e$. 
For $g \in C_{0}(S)$,

$$
\begin{aligned}
\int g d \mu\left(x,\left[\left(1_{e_{2}} \otimes y\right), g^{\prime}\right]\right) & =\left[\left(1_{e_{2}} \otimes y\right), g^{\prime}\right](g \cdot x) \\
& =\left(1_{e_{2}} \otimes y\right)\left(\left\langle g^{\prime}, g \cdot x\right\rangle\right)=\mu\left(y,\left\langle g^{\prime}, g \cdot x\right\rangle\right)\left(e_{2}\right), \\
\int g d \nu\left(x y, g^{\prime}\right) & =\int g \chi_{e_{2}} d \mu\left(x y, g^{\prime}\right)=\int_{e_{2}} g d \mu\left(x y, g^{\prime}\right)=w\left(e_{2}\right) .
\end{aligned}
$$

For $h \in C_{0}(S)$,

$$
\begin{gathered}
\int h d \mu\left(y,\left\langle g^{\prime}, g \cdot x\right\rangle\right)=\left\langle g^{\prime}, g \cdot x\right\rangle(h \cdot y)=g^{\prime}(g h \cdot x y), \\
\int h d w=\int h g d \mu\left(x y, g^{\prime}\right)=g^{\prime}(h g \cdot x y) .
\end{gathered}
$$

This completes the proof of the lemma.

Proof of Theorem 2.1. Suppose for $f, g \in C_{0}(S, A), T(f g)=T(f) T(g)$. By Proposition 2.2 and Lemma 2.3, for $e_{1}, e_{2} \in \mathscr{B}(S)$,

$$
\begin{aligned}
K\left(e_{1} \cap e_{2}\right)(x y) & =T^{\prime \prime}\left(1_{e_{1} \cap e_{2}} \otimes x y\right)=T^{\prime \prime}\left(1_{e_{1}} \otimes x\right)\left(1_{e_{2}} \otimes y\right) \\
& =T^{\prime \prime}\left(1_{e_{1}} \otimes x\right) T^{\prime \prime}\left(1_{e_{2}} \otimes y\right)=\left(K\left(e_{1}\right) x\right)\left(K\left(e_{2}\right) y\right)
\end{aligned}
$$

Now suppose $K\left(e_{1} \cap e_{2}\right)(x y)=\left(K\left(e_{1}\right) x\right)\left(K\left(e_{2}\right) y\right)$ for $e_{1}, e_{2} \in \mathscr{B}(S), x, y \in A$. If $f=\chi_{e_{1}} \cdot x, g=\chi_{e_{2}} \cdot y$, then,

$$
\begin{aligned}
\int f g d K & =\int \chi_{e_{1} \cap e_{2}} x y d K=K\left(e_{1} \cap e_{2}\right) x y \\
& =\left(K\left(e_{1}\right) x\right)\left(K\left(e_{2}\right) y\right)=\left(\int f d K\right)\left(\int g d K\right) .
\end{aligned}
$$

For arbitrary $f, g \in C_{0}(S, A)$, a routine argument as in the last part of the proof of [10, Theorem 1] shows that $\int f g d K=\left(\int f d K\right)\left(\int g d K\right)$ and this completes the proof of the theorem.

3. Involution preserving operators. In this section we assume that $A, B$ are Banach algebras with isometric involution *. For $f \in C_{0}(S), x \in A$, we define $(f(s) \cdot x)^{*}$ $=\overline{f(s)} \cdot x^{*}$, where the bar denotes the complex conjugate. For $f \in C_{0}(S, A), f^{*}$ is defined in the natural way by taking the limit process. Recall that involutions could be defined in $A^{\prime}$ and $A^{\prime \prime}$ in a natural way (see, for example, Bonsall and Duncan [3]).

Lemma 3.1. For $x \in A, e \in \mathscr{B}(S),\left(1_{e} \otimes x\right)^{*}=\left(1_{e} \otimes x^{*}\right)$.

Proof. For $f^{\prime} \in C_{0}^{\prime}(S, A)$,

$$
\begin{aligned}
& \left(1_{e} \otimes x^{*}\right)\left(f^{\prime}\right)=\mu\left(x^{*}, f^{\prime}\right)(e), \\
& \left(1_{e} \otimes x\right)^{*}\left(f^{\prime}\right)=\left(\left(1_{e} \otimes x\right)\left(\left(f^{\prime}\right)^{*}\right)\right)^{*}=\left(\mu\left(x,\left(f^{\prime}\right)^{*}\right)(e)\right)^{*} .
\end{aligned}
$$


For $f \in C_{0}(S)$,

$$
\begin{aligned}
\int f d \mu\left(x^{*}, f^{\prime}\right) & =f^{\prime}\left(f \cdot x^{*}\right), \\
\int f d \bar{\mu}\left(x,\left(f^{\prime}\right)^{*}\right) & =\left(\int \bar{f} d \mu\left(x,\left(f^{\prime}\right)^{*}\right)\right)^{*} \\
& =\left(\left(f^{\prime}\right)^{*}(\bar{f} \cdot x)\right)^{*}=f^{\prime}\left(f \cdot x^{*}\right),
\end{aligned}
$$

and the lemma is proved.

Proposition 3.2. Let $T: A \rightarrow B$ be a bounded linear operator. Then the operator $T$ satisfies $T\left(f^{*}\right)=(T(f))^{*}$ for all $f \in A$ iff $T^{\prime \prime}\left(F^{*}\right)=\left(T^{\prime \prime}(F)\right)^{*}$ for all $F \in A^{\prime \prime}$.

Proof. We only prove the necessity. For $b^{\prime} \in B^{\prime}$,

$$
\begin{aligned}
T^{\prime \prime}\left(F^{*}\right)\left(b^{\prime}\right) & =F^{*}\left(T^{\prime}\left(b^{\prime}\right)\right), \\
\left(T^{\prime \prime}(F)\right)^{*}\left(b^{\prime}\right) & =\left(T^{\prime \prime}(F)\left(b^{\prime}\right)^{*}\right)^{*}=\left(F\left(T^{\prime}\left(\left(b^{\prime}\right)^{*}\right)\right)\right)^{*} \\
& =F^{*}\left(\left(T^{\prime}\left(\left(b^{\prime}\right)^{*}\right)\right)^{*}\right) .
\end{aligned}
$$

For $f \in A$,

$$
\begin{aligned}
\left(T^{\prime}\left(\left(b^{\prime}\right) *\right)\right)^{*}(f) & =\left(T^{\prime}\left(\left(b^{\prime}\right) *\right)\left(f^{*}\right)\right)^{*}=\left(\left(b^{\prime}\right) *\left(T\left(f^{*}\right)\right)\right)^{*} \\
& =\left(\left(b^{\prime}\right) *\left((T(f))^{*}\right)\right)^{*}=b^{\prime}(T(f)) .
\end{aligned}
$$

This completes the proof of the proposition.

We are now ready to prove the following theorem, which shows that the involution preserving property of an operator is reflected naturally in its representing measure.

THEOREM 3.3. The operator $T$ satisfies $T\left(f^{*}\right)=(T(f))^{*}$ for all $f \in C_{0}(S, A)$ iff $(K(e) x)^{*}=K(e) x^{*}$ for all $e \in \mathscr{B}(S), x \in A$.

Proof. If $T\left(f^{*}\right)=(T(f))^{*}$ then, by Proposition 3.2, Lemma 3.1, $(K(e) x)^{*}=$ $\left(T^{\prime \prime}\left(1_{e} \otimes x\right)\right)^{*}=T^{\prime \prime}\left(1_{e} \otimes x^{*}\right)=K(e) x^{*}$.

On the other hand suppose $(K(e) x)^{*}=K(e) x^{*}$ for all $e \in \mathscr{B}(S), x \in A$. If $f=\chi_{e} \cdot x$, then $f^{*}=\chi_{e} \cdot x^{*}$ and

$$
\left(\int x_{e} \cdot x d K\right)^{*}=(K(e) x)^{*}=K(e) x^{*}=\int x_{e} \cdot x^{*} d K .
$$

For $f \in C_{0}(S, A)$, a routine argument again shows that $(T(f))^{*}=T\left(f^{*}\right)$.

We conclude this paper with a remark that results of this paper may be used to prove the Spectral Theorem for bounded operators (see Johnson [9, p. 99]).

\section{REFERENCES}

1. R. G. Bartle, A general bilinear integral, Studia Math. 15 (1956), 337-352.

2. R. Bartle, N. Dunford and J. Schwartz, Weak compactness and vector measures, Canad. J. Math. 7 (1955), 289-305.

3. F. F. Bonsall and J. Duncan, Numerical ranges of operators on normed spaces and of elements of normed algebras, London Math. Soc. Lecture Note Series, vol. 2, Cambridge Univ. Press, London and New York, 1971.

4. J. K. Brooks and P. W. Lewis, Linear operators and vector measures, Trans. Amer. Math. Soc. 192 (1974), 139-162. 
5. S. T. L. Choy, Riesz representation theorem and weak compactness, Anal. Math. 6 (1980), 199-205.

6. N. Dinculeanu, Vector measures, Internat. Series of Monographs in Pure and Appl. Math., vol. 95, Pergamon Press, New York; VEB Deutscher Verlag der Wissenschaften, Berlin, 1967.

7. J. Gil de Lamadrid, Measure and tensors, Trans. Amer. Math. Soc. 114 (1965), 98-121.

8. R. K. Goodrich, A Riesz representation theorem, Proc. Amer. Math. Soc. 24 (1970), 629-636.

9. G. W. Johnson, Integral representations of certain linear operators, Thesis, University of Minnesota, 1968.

10. Integral representation of multiplicative, involution preserving operators in $\mathfrak{L}(C(S), E)$, Proc. Amer. Math. Soc. 23 (1969), 373-377.

Department of Mathematics, National University of Singapore, Republic of Singapore 1025 